\title{
Diez tesis para una teoría de las Relaciones Transestatales Félix Pablo Friggeri*
}

\section{RESUMEN}

Este trabajo tiene como objetivo presentar una propuesta básica para una agenda de investigación con miras a una construcción teórica que aporte a una perspectiva popular y descolonizadora en el ámbito de las habitualmente llamadas teorías de las Relaciones Internacionales. Proyectando hacia este ámbito el principio cosmovisional y constitucional de la plurinacionalidad se presenta un cuestionamiento a una serie de elementos de las teorías predominantes y se busca comenzar a colocar las bases para caminar en un proceso de investigación y análisis hacia una construcción teórica alternativa que, provisoriamente, se denomina teoría de las Relaciones Transestatales. Metodológicamente, se trata de un trabajo interdisciplinario y que trabaja la articulación de conceptos en camino a una teoría, como lo propusiera Amado Cervo, donde se trabaja en base a propuestas surgidas de tres fuentes: desde la reflexión de las luchas populares de la región, principalmente de las provenientes de los movimientos indígenas; desde conceptos y convicciones surgidas de la historia regional y su reflexión; $y$, desde la base, principalmente de autores latinoamericanos que han trabajado la misma perspectiva popular y descolonizadora que pretende tener el artículo. Desde allí se elaboran diez tesis con la intención de que sirvan de base para una articulación teórica. La estructuración del trabajo se hace en relación a la formulación y explicación de estas diez tesis. Esta explicación no llega a ser exhaustiva por la limitada extensión que debe tener un artículo de estas características, pero también, por lo menos en algunos casos, por tratarse de un proceso todavía en construcción y que necesita continuar su profundización. De todas formas, se aspira a que la presentación conjunta de estas tesis proporcione una visión panorámica que posibilite visualizar los contenidos fundamentales del proyecto teórico. Existen también una serie de temas cuyo tratamiento podría complementar y enriquecer la propuesta, y que no son tratados en el artículo por los límites expuestos más arriba. Las temáticas abordadas a través de las tesis son resumidamente las siguientes: I) Desde el concepto de plurinacionalidad se propone el de relaciones transestatales. 2) Se propone esta teoría como paradigma distinto al de las teorías de las Relaciones Internacionales predominantes. 3) La integración desde los pueblos. 4) Las praxis de las luchas populares como lugar epistémico político. 5) Soberanía popular y soberanía compartida. 6) El análisis del racismo de clase. 7) La integración contrahegemónica. 8) El aporte al diálogo Sur Sur. 9) La búsqueda de un horizonte poscapitalista. I0) La visión biocéntrica.

\section{Palabras Clave}

Teoría de las Relaciones Transestatales; praxis de lucha popular; integración latinoamericana y caribeña; Plurinacionalidad; Soberanía Popular; Relaciones Internacionales; lugar epistémico político.

\section{TitLe}

Ten Theses for a Theory of Trans-State Relations

\section{Extended Abstract}

The objective of this paper is to present a basic proposal for a research agenda with a view to a theoretical construction that contributes to a popular and decolonizing perspective in the field of what is usually called Theories of International Relations. Projecting the cosmovisional and constitutional principle of Plurinationality towards this area, a questioning of a series of elements of the predominant theories is presented. Moreover, it seeks to begin to lay the foundations to pursue a process of research and analysis towards an alternative theoretical construction that, provisionally, is called Theory of Trans-State Relations. The epistemic-political basis of it is the reflection on the praxis of popular struggle and its potential to become an analytical perspective for trans-state relations. Methodologically, it is an interdisciplinary work that works on the articulation

DOI:

https://doi.org/I0.15366/relacionesinternacionales202I.48.002

Formato de citación recomendado:

FRIGGERI, Félix Pablo (202I). “Diez tesis para una Teoría de las Relaciones Transestatales”, Relaciones Internacionales, $\mathrm{n}^{\circ}$ 48, pp. 3I-50.
* Félix Pablo FRIGGERI, Profesor Adjunto en el Área de Relaciones Internacionales e Integración; Coordinador de la Maestría en Integración Contemporánea Latinoamericana; Coordinador Local del Dinter con PUC-Río; Director del Instituto Latinoamericano de Economía, Sociedad y Política (20I3-20I7).

Contacto:fpfriggeri@ hotmail.com

Recibido: 22.02.202I

Aceptado: |8.08.202| 
of concepts on the way to a theory, as proposed by Amado Cervo, where it works based on proposals arising from three sources: from the reflection of the popular struggles of the region, mainly from those coming from indigenous movements; from concepts and convictions arising from regional history and its reflection; and, from the base, mainly, of Latin American authors who have developed the same popular and decolonizing perspective that the article claims to have. From there, ten theses are prepared with the intention that they serve as the basis for a theoretical articulation. The structuring of the work is done in relation to the formulation and explanation of these ten theses. This explanation is not exhaustive due to the limited extension that an article of these characteristics must have. However, also, at least in some cases, because it is a process still under construction and that needs to be developed further. In any case, it is hoped that the joint presentation of these theses provides a panoramic vision that makes it possible to visualize the fundamental contents of the theoretical project. There are also a series of topics whose treatment could complement and enrich the proposal, and which are not treated in the article due to the limits set forth above.

In the introduction, a brief presentation is made of the historical and current problems of academic production on the socalled Theories of International Relations, noting that it predominantly contains a character strongly linked to the interests and perspectives of the main sectors of power worldwide. Their colonized reproduction in our region causes a predominant environment which engenders a lack of creativity and, above all, lack of reference to the realities of our peoples and their struggles.Although there are invaluable efforts to build alternatives closer to our realities, they are still limited in number and, many times, politically and epistemically limited.

The ten proposed theses are the following:

The Latin American and Caribbean States are Plurinational - even though only two have constitutionally recognized it therefore the relations that transcend the States are not international relations but trans-state relations

The colonized character of the Theory of International Relations makes it fundamentally useless and harmful for an analysis of the relations that transcend the State from a Latin American popular perspective, therefore new paradigms must be created for its reading: the Theory of Trans-State Relations aspires to be one of them

The Theory of Trans-State Relations provides elements to overcome the state-centricity that marked the evolution of the study of so-called "international relations", focusing on the issue of integration from the peoples of the region.

The Theory of Trans-State Relations intends to make an epistemic contribution to the study of relations that transcend the States in Latin America and the Caribbean based on the production of knowledge from popular, mainly indigenous, wisdom in the fight against colonial-capitalist imposition

The Theory of Trans-State Relations maintains that Popular Sovereignty must be distinguished and repositioned above State Sovereignty. Therefore, it must also be its guide and reference, and in order to strengthen both dimensions of Sovereignty in Latin America and the Caribbean, they have to find ways to exercise Shared Sovereignty among the peoples and states of the region.

The Theory of Trans-State Relations argues that it is necessary through construction of knowledge to provide an analysis of Class Racism that goes through the so-called "international relations"

The Theory of Trans-State Relations intends to provide knowledge that gives consistency and validity to the counterhegemonic integration process of the Latin American and Caribbean region.

The Theory ofTrans-State Relations, thought from Latin America, can be a contribution to the South-South dialogue since its decolonizing approach in the epistemic and the political points to a common reality of the Political South

The Theory of Trans-State Relations starts from a commitment to a search for paths of justice and alternative paths to overcome capitalism that, based on the praxis and reflection of the Indigenous Movement, recovers in a fruitful Dialogue of Knowledge the great Latin American contributions in this sense (Theory of Dependency, Liberation Christianity, IndoAmerican Socialism, and all the reflection and the original revolutionary praxis very rich in Latin America). Thus, it aspires to serve as a joint contribution, mainly with the other regions affected by the Coloniality of Power (or of the Political South) so that this search can be global

The Theory of Trans-State Relations works from a biocentric paradigm, based on the worldviews and praxis of indigenous peoples and nations, which must be fundamental elements for the articulation of relations between peoples and between States.

Each of these theses is accompanied by an explanatory analysis where some of the bases of its support are proposed.As already stated, the analysis in this article is not exhaustive, and aims to provide elements to constitute a panoramic vision that allows the basic understanding of the meaning of the theoretical proposal that is being built.

In the final considerations, together with the review of the central issues, the intention is expressed to broaden the agenda of the so-called "international relations", but also to generate debate on the issues that habitually and currently occupy it from a popular and decolonizing Latin American-Caribbean perspective. The conviction is also expressed that this proposal can be projected towards dialogue with other projects of the Political South. Finally, the need to link the popular struggles of the region with the task of producing academic knowledge is affirmed.

\section{KEYWORDS}

Trans-State Relations Theory; praxis of popular struggle; Latin American and Caribbean integration; Plurinationality; Popular sovereignty; International relations; epistemic-political place. 


\section{ntroducción}

La teoría de las Relaciones Internacionales viene siendo elaborada, fundamentalmente, desde sectores de poder mundial y de una academia ligada a ellos. Su conformación predominante en América Latina mantiene una adhesión general a esta tendencia y configura, dentro de las ciencias sociales, un caso extremo de eurocentrismo tanto en su orientación política como en sus fuentes epistémicas.

Se han venido realizando algunos valiosos esfuerzos para revertir esta situación. Pero hay que reconocer que todavía son limitados en número y en alcance temático y, más todavía, en lo que podemos llamar alcance epistémico.

Presento aquí un aporte desde un planteo epistémico político latinoamericano, popular y descolonizador. Lo hago en forma de diez tesis como base de una agenda de investigación buscando una articulación de conceptos y perspectivas que puedan constituir el camino hacia una teoría, todavía abierta y en proceso.

El punto de partida es el principio de plurinacionalidad. Proclamado en las Constituciones de Ecuador —en 2008- y Bolivia —en 2010 - al declarar a estos países como estados plurinacionales. Fruto, fundamentalmente, del pensamiento y la praxis de sus movimientos indígenas, este hecho constituye el más profundo replanteo del estado nación latinoamericano en toda su historia. Repensar el estado nos lleva necesariamente a repensar lo que se ha venido llamando — desde un imaginario coherente con esos estados nación- Relaciones Internacionales y las teorías que pretenden explicarlas y analizarlas.

Propongo aquí lo que denomino teoría de las Relaciones Transestatales. Ésta, todavía en construcción, debe trabajarse, profundizarse y ampliarse. En esta presentación intento articular un conjunto de conceptos y perspectivas en la línea propuesta en forma abarcativa y panorámica. Al darle carácter de agenda básica tiene el costo de que el tratamiento de los distintos aspectos no llega a ser exhaustivo, lo cual proyecta su profundización hacia trabajos posteriores.

Entre los elementos aquí tratados que componen la propuesta están: el sentido de la transestatalidad; la superación del estadocentrismo; la integración regional contrahegemónica y desde los pueblos; la primacía de la soberanía popular sobre la estatal; la praxis de lucha popular como lugar epistémico político y la posibilidad de articulación de sus sujetos; su proyección hacia el Sur Político; el racismo de clase; el biocentrismo desde la propuesta del Buen Vivir. Trabajo fundamentalmente, por opción, con autores latinoamericanos, aunque también con otros del Sur Político y de los que trabajan en espacios hegemónicos para establecer puntos de diálogo con el debate que se realiza en este ámbito. 


\section{Los estados latinoamericanos y caribeños son plurinacionales -aun cuando solamente dos lo han reconocido constitucionalmente- por tanto las relaciones que trascienden a los estados no son relaciones internacionales sino relaciones transestatales}

El proceso de constitución de los estados de nuestra región estuvo marcado por la artificialidad y la incompletud presuponiendo una correlación con una impuesta identidad nacional. Esa falta de correspondencia con la realidad se evidenció, sobre todo, en algunos países de fuerte presencia comunitaria indígena en número, pero sobre todo en calidad de organización y movilización. La reflexión política y epistémica de estos movimientos mostró que todos los estados latinoamericanos son plurinacionales aunque su composición étnica y sus procesos históricos sean relativamente distintos.

Proyectando esta reflexión a las relaciones que trascienden los estados se impone repensar los planteos básicos de lo que se dio en llamar Relaciones Internacionales y las teorías que pretenden analizarlas. Entre los autores latinoamericanos recientes, Marcelo Gullo (2017, p. 13) resalta esta realidad de estados formados por distintas naciones, y aunque cuestiona la "nominación" de las "Relaciones Internacionales", opta "por razones de orden práctico" por no "crear un nuevo nombre para nuestra disciplina de estudio". Propongo, en cambio, partiendo desde el principio de plurinacionalidad y para una mayor correspondencia con la realidad, que estas relaciones sean denominadas transestatales.

Soy consciente que el solo nombre de teoría de las Relaciones Transestatales no describe todo lo que pretende ser su contenido. Su significación gana en riqueza si recordamos que su punto de partida es el proceso, con protagonismo indígena, que generó el principio de plurinacionalidad. Ese proceso aporta al contenido latinoamericano, popular y descolonizador de esa denominación.

La historia y la realidad actual de la lucha de los pueblos y naciones indígenas evidencian dos formas de transestatalidad. Una forma en que trascienden el estado es diacrónica y está dada por la preexistencia de estos pueblos y naciones a la conformación de los estados nación. Esto tiene importantes consecuencias en el análisis histórico y jurídico y en la estructuración de la institucionalidad política. Los trascienden porque son anteriores a ellos. La otra forma es sincrónica ya que actualmente muchas naciones indígenas tienen repartida su población en distintos estados. Ambas formas deben ser tenidas en cuenta a la hora de analizar las relaciones transestatales.

2. El carácter colonizado de la teoría de Relaciones Internacionales la hace fundamentalmente inútil y nociva para un análisis de las relaciones que trascienden al estado desde una óptica popular latinoamericana, por tanto deben ser creados nuevos paradigmas para su lectura: la teoría de las Relaciones Transestatales pretende ser uno de ellos

Mucho se ha escrito en torno al yanquicentrismo de las teorías de Relaciones Internacionales (Cepeda M.et al.,2016,p.54).Es un problema político y a la vez, desde la producción de conocimiento, un problema epistémico. Expresa tanto la colonialidad del poder como la colonialidad del saber. Lo 
más grave no es que los centros de elaboración tengan poca o nula recepción a un pensamiento otro surgido del Tercer Mundo y, quizás, menos todavía, de América Latina. Lo más grave es que la investigación y la docencia sobre el tema en nuestra región siga centrada en esa producción colonizada e imperialista. Necesitamos la creación de teorías y metodologías propias. Hay avances críticos pero todavía hay pocas alternativas planteadas desde una originalidad epistémica y desde una búsqueda política autónoma. En este sentido la teoría de las Relaciones Transestatales pretende aportar desde la convicción de que una teoría construida en la región "no puede estar separada del contexto histórico, político e intelectual de América Latina” (Briceño R. y Simonoff, 2017, p. 43).

La misma academia latinoamericana muchas veces se limita a reflejar en sus debates lo que se piensa en otros lugares no solo analizando problemáticas ajenas a la región sino, lo que es peor, consolidando la dominación que nos afecta. Así "los expertos en Relaciones Internacionales en México, en Buenos Aires, en Río de Janeiro [...] reflejan y siguen, más o menos servilmente y con algún retraso, las 'modas' norteamericanas — los debates y las categorías de análisis en boga - al hacerlo, reflejan, y sirven también, muy a menudo, al interés político de los Estados Unidos” (Gullo, 2017, p. 6). Así la disciplina devino en un lugar privilegiado donde se transforman "los intereses hegemónicos en conocimientos verdaderos" (Cairo y Bringel, 20I0, p. 45).

A pesar de todo el proceso de reconstrucción epistémica que acompañó la lucha contra el neoliberalismo y la reflexión sobre los quinientos años de la invasión europea a América, en las Ilamadas Relaciones Internacionales los cambios fueron pocos y lentos. Arlene Tickner (2003, p. 297) planteaba que a comienzos del siglo los cambios, tanto en las publicaciones como en la orientación de la enseñanza, eran "sorprendentemente" pocos. Hay esfuerzos importantes. Algunos vienen desde el corazón disciplinar, como el que ya hace un tiempo planteara Amado Luiz Cervo $(2003,2008)$, otros de un diálogo con otras disciplinas.

La clave para una teoría de las Relaciones Transestatales es abrir y consolidar una agenda popular en el estudio de las llamadas Relaciones Internacionales y establecer un enfoque epistemológico alternativo de análisis desde las luchas de nuestros pueblos. La superación popular y latinoamericana de la agenda y de la perspectiva hegemónica es su objetivo. No basta solamente la crítica a las teorías de las Relaciones Internacionales (Hobson y Sajed, 20I 7, p. 548), se necesita, además, abrir caminos a la creatividad de la propuesta, a la "creación heroica" desde las luchas populares latinoamericanas.

\section{La teoría de las Relaciones Transestatales brinda elementos para superar el estadocentrismo que marcó la evolución del estudio de las llamadas Relaciones Internacionales centralizándose en el tema de la integración desde los pueblos de la región}

La búsqueda de una perspectiva popular en las relaciones que trascienden al estado tiene que plantear la superación de su centralidad absoluta y poder analizar la realidad, las potencialidades y las dificultades de las relaciones entre los pueblos y su potencial de integración. 
En las reflexiones latinoamericanas recientes de las llamadas Relaciones Internacionales se ha planteado tanto superar el papel del estado nación como "átomo epistemológico, teórico y metodológico" (Soto Acosta, 2015, p. 26), como la necesidad de "incluir a las masas en el estudio de las Relaciones Internacionales” (Tickner, Cepeda y Bernal, 20I5, p. 806)'. Al analizar el pensamiento académico regional sobre Relaciones Internacionales, Eduardo Devés-Valdés (2013, p. 58) se preguntaba por el camino para descentrar la reflexión del estado y buscar "otra unidad de análisis" porque era "imposible" pensar en un mundo mas igualitario desde los "prejuicios" de las "Relaciones Internacionales".

Propongo una trascendencia al estado desde lo popular, desde abajo. La teoría de las Relaciones Internacionales hegemónica había planteado una trascendencia desde los grandes poderes económicos. Es el debate sobre los "actores" (Gullo, 2017, pp. 17-18). Afirmar la centralidad de los pueblos como sujetos de las relaciones y de la integración latinoamericana no significa prescindir de los estados. Se trata de superar un estadocentrismo ensimismado. Aunque los estados latinoamericanos conservan, y lamentablemente muchas veces hacen honor, a su carácter de instrumento de la clase oligárquica en nuestra región, también es cierto que los procesos más profundos de democratización política, económica y cultural en América Latina se dieron cuando el estado asumió la función de poner límites al mercado. Por eso, desde las luchas populares no se puede pensar la profundización democratizadora y de ejercicio de la soberanía popular sin el estado.

Mirándolo desde la integración latinoamericana, ésta solamente tiene sentido si plenifica, enaltece, enriquece y hace más justa la vida de los pueblos que componen el subcontinente. En la Carta de los Movimientos Sociales de las Américas hacia el Alba en el Foro Social Mundial de Belem de Pará se decía:

“[...] nuestro horizonte fundamental es la integración desde abajo, a partir de los pueblos, para impulsar transformaciones antineoliberales $y$ antiimperialistas, que rescaten las formas alternativas y solidarias de intercambio que hemos creado en nuestros territorios para enfrentar la dominación neoliberal. La memoria y las luchas que hemos compartido se convierten en un acumulado de fuerzas que sostienen este sueño de unidad por la construcción de un continente justo, libre de hegemonías imperialistas, de poderes patriarcales y donde germina el buen vivir entre los pueblos" (Asamblea de los Movimientos Sociales, 2009).

La articulación de los movimientos sociales populares es clave en esta búsqueda. Es un tema que conviene relacionar con el más tradicional del internacionalismo proletario. Sobre todo pensando en una redefinición de lo que es el proletariado en América Latina, como sujeto político y como sujeto epistémico. Aquí la búsqueda de José Carlos Mariátegui puede aportar muchísimo,

Existe una relación entre varios elementos expuestos en este artículo y los que realizó Robert W. Cox (20I4): la "pluralidad de formas de estado"; la construcción de teorías desde una "perspectiva" o "punto de vista”; el hecho de caracterizar la "teoría crítica" como "teoría de la historia"; la necesidad de que la investigación en este ámbito vaya "más allá de las Relaciones Internacionales convencionales"; la crítica al "castigo" de "otras opciones de pensamiento”; el análisis de "una contrahegemonía basada en una coalición del Tercer Mundo". 
con su ampliación del proletariado y su centramiento en lo indígena (Flores G. 2008, pp. 26236I). Esta articulación puede dinamizar la perspectiva propuesta por su alternatividad al sistema dominante. Por este carácter son importantes especialmente las organizaciones indígenas y campesinas, las cuales ya cuentan con vínculos regionales funcionando. Pero también sindicatos, centrales obreras, intelectuales, artistas, comunicadores, todos pueden hacer un aporte valioso en este sentido.

Esta articulación organizada de los movimientos populares requiere de un apoyo estatal que no les haga perder autonomía ${ }^{2}$.También la creación y consolidación de espacios institucionalizados que puedan facilitar tanto el apoyo logístico como el político. Existen experiencias históricas en las cuales conviene profundizar. Por nombrar dos originales: una es la de la comunicación entre las escuelas rurales indígenas que articuló el pensamiento de la educación socialista con el de la escuela ayllu y que unió a dos instituciones emblemáticas como son la de Ayotzinapa en México con la de Warisata en Bolivia en los años treinta y cuarenta. Otra la experiencia del agregado obrero en las embajadas argentinas del gobierno del primer peronismo que hacía el contacto con los movimientos obreros de otros países.

En el proceso más reciente la experiencia del Mercosur social y solidario estaba marcada por esa idea, como también el concepto de diplomacia de los pueblos que se planteó en la política exterior de los gobiernos de Evo Morales (Ticona Alejo, 2016, p. 42). La más clara fue la de la Alianza Bolivariana para los Pueblos de Nuestra América (ALBA) (Stédile, Piñero y Bertoldi, 20I4, p. I35) como "integración regional de base" (Díaz M., 20I4) y que fue valorada también en otros lugares (Al-Kassimi, 20I8)3.

El otro espacio clave son las áreas de frontera. Desde las ideologías conformadoras del estado nación, las fronteras fueron pensadas como separación de los pueblos y como resguardo de la actividad del capital hacia dentro. Sin embargo, los pueblos las vivieron predominantemente como espacios de encuentro y articulación. Lo hacen a pesar de los estados, pero tendría que hacerse con el apoyo decidido de los estados. Los espacios de frontera tienen que ser pensados como espacios de unión de los pueblos que viven en sus cercanías y como espacios puentes de la unidad latinoamericana y caribeña.

4. La teoría de las Relaciones Transestatales pretende hacer un aporte epistémico al estudio de las relaciones que trascienden a los estados en América Latina y el Caribe basado en la producción de conocimiento desde las sabidurías populares, principalmente indígenas, en lucha contra la imposición colonial capitalista

No se puede reflexionar, analizar y construir conocimiento que acompañe una integración de este tipo (popular, contrahegemónica) desde los parámetros coloniales y capitalistas. Se requiere

\footnotetext{
El tema de las relaciones entre el estado y los movimientos populares es abordado, para el caso de los piqueteros en Argentina, por Svampa y Pereyra (2009) y Dinerstein (2010), con reflexiones que pueden servir para comprender la complejidad de las mismas.

3 Uno de los espacios que estuvo ligado al proceso de la ALBA fundamentalmente y que trabaja en un importante proceso de articulación entre movimientos es Vía Campesina, y su organización regional la CLOC (Coordinadora Latinoamericana de Organizaciones del Campo). Animador fundamental de este espacio es el Movimento dos Trabalhadores Rurais Sem Terra (MST) y la escuela Florestan Fernandes ligada a este movimiento.
} 
un proceso de descolonización descapitalización del conocimiento, pero también la capacidad de construcción de un pensamiento alternativo y original latinoamericano. Para eso entiendo importante resaltar, principalmente, dos temas claves: el del lugar epistémico político desde donde se construye el conocimiento y el de las fuentes del mismo. Dos temas fuertemente interconectados pero que conviene, en principio, distinguir.

El lugar desde donde se produce conocimiento está más en el corazón de la persona o del colectivo que en la determinación geográfica, aunque también ésta es importante. Es una opción de compenetración. El hacer esa opción implica también asumir un lugar de percepción. Es que ese lugar determina las lejanías y las cercanías, desde allí uno escucha, ve, intercambia, aprende y propone. Así el lugar acerca o aleja de distintas realidades y de los conocimientos elaborados en ellas. Por esto ese lugar, además de epistémico, es también afectivo, ético y político. Esto vale para todos los productores de conocimiento, también para todos los del mundo académico. Pero habitualmente los que están comprometidos con el conocimiento dominante niegan o invisibilizan ese lugar. Todos producen conocimiento estando cerca o lejos de determinados colectivos, intereses, ideas, propuestas, luchas. Esto hace que la neutralidad sea imposible $y$, siendo invocada como argumento, falsa, tanto afectiva y ética, como política y epistémicamente.

Lo primero para construir un conocimiento latinoamericano es esta opción por el lugar desde donde producirlo. Uno de los primeros espacios donde se trató sólidamente este tema fue el de la Filosofía y la Teología de la liberación latinoamericanas. Rodolfo Kusch (I978, p. 73) sostenía que había que "replantear el problema del pensamiento desde el subsuelo mismo de nuestra sociedad”. El emblemático vasco salvadoreño Ignacio Ellacuría (2005) realizaba una propuesta de "lugar teológico" que es, entiendo, perfectamente traducible como lugar epistémico político desde donde se realiza la praxis de lucha, la reflexión sobre la misma y, por tanto, la construcción de saberes. Para él hay dos lugares epistemológicos que no pueden separarse y están en relación dialéctica:"América Latina” y"los pobres” o, en algunos párrafos, “la lucha de los pobres". Solamente "en relación con los pobres [...] se entiende qué es América Latina como lugar teológico". También sostiene Ellacuría $(2005$, p. 9) que el lugar es "desde donde se hace la vivencia y la reflexión". La palabra desde es clave en la construcción del conocimiento indicando tanto el origen como la direccionalidad que asume. Los pobres y sus luchas son determinantes para pensar América Latina como lugar epistémico político. Por eso el lugar no es cualquier América Latina, son fundamentales los lugares de los más latinoamericanos de los latinoamericanos: el mundo indígena y el mundo popular, especialmente el campesino. Quedan fuera los lugares que aún situados geográficamente en la región están marcadamente eurocentrados: todo tipo de oligarquías, entre ellas la académica.

Este lugar da la posibilidad de una crítica profunda frente a la explotación y dominación, a lo que podríamos llamar el empobrecimiento de los pobres. Pero, sobre todo, da la posibilidad de referirse a la inmensa riqueza de sabiduría que existe en su lucha, por eso, además de la crítica, capacita para las propuestas alternativas, contrahegemónicas. Se puede relacionar esto con el concepto de Dussel (2007, pp. 347-349) de "exterioridad" del pobre respecto al sistema imperante y a su "afirmación" en cuanto "Otro como otro, no como lo mismo"4. Al proponer

También Rocha y Maso (2020, p. 15), desde los estudios para la paz, proponen "la perspectiva de la alteridad" como "ampliación de fronteras de las Relaciones Internacionales". 
la "liberación" como referencialidad clave de su planteo, la define como "tener la creatividad de ser realmente capaces de construir la novedad, un nuevo momento histórico desde la positiva exterioridad cultural de nuestro pueblo" (Dussel, 1977, p. 69).

El hecho de caracterizar al lugar como epistémico político ${ }^{5}$ está relacionado con el proceso realizado especialmente por los movimientos indígenas andinos, que se denominó la politización de lo étnico ${ }^{6}$. Expresa, por un lado, que el compromiso transformador en lo político está unido a una reconstrucción del conocimiento (Macas, 2005). Por otro lado expresa la negación de la supuesta neutralidad científica que opera como legitimación de la pretensión de universalidad del discurso dominante.

Esto se relaciona con el planteo de "colonialidad del saber" derivado de lo que Aníbal Quijano (2014) expuso como colonialidad del poder. Entendido como "el posicionamiento del eurocentrismo como la perspectiva única del conocimiento, la que descarta la existencia y viabilidad de otras racionalidades epistémicas y otros conocimientos que no sean los de los hombres blancos europeos o europeizados" (Walsh, 2008, p. 135). Presupuesto clave para entender una actitud de "servilismo intelectual" que se expresa en "la adopción casi ciega de los modelos teóricos y conceptos desadaptados a nuestro medio, pero que tienen sus referentes en Europa y los Estados Unidos” (Fals B., 2009, p. 224). El carácter dialéctico y político que se otorga a este lugar de conocimiento (Ellacuría, 2005) habilita a pensar una "insurgencia epistémica" dada, principalmente, por la praxis y la reflexión de los "movimientos sociopolíticos ancestrales" y su capacidad de proponer "lógicas, racionalidades y conocimientos distintos" (Walsh, 2008, p. I34).

Desde la temática de las Relaciones Internacionales, Gullo (2017, pp. I0-I I) sostiene que "el lugar desde donde miramos y observamos, genera categorías" y propone - tomando un concepto del filósofo argentino Mario Casalla - "pensar desde lo 'universal situado'” y construir "un corpus epistémico" que "permita dar cuenta de nuestra perspectiva”. La conciencia, la decisión y la praxis política de asumir un lugar comprometido con las mayorías populares dan la capacidad reflexiva y propositiva de enfrentar la pretensión de universalidad del pensamiento moderno colonial con propuestas latinoamericanas.

Queda propuesto, entonces, como lugar epistémico político desde donde construir el conocimiento original y latinoamericano para una teoría de las relaciones estatales: la praxis de lucha popular por la vida en todas sus manifestaciones. Pasando a las fuentes conviene aclarar que es un tema muy ligado con el del lugar aunque distinguible analíticamente: "la distinción no es estricta, ni menos excluyente, porque de algún modo el lugar es fuente, en cuanto aquél hace que ésta dé de sí esto o lo otro, de modo que gracias al lugar y en virtud de él se actualizan y se hacen realmente presentes unos determinados contenidos" (Ellacuría, 2005, p. 9). Las fuentes dan la materialidad al pensamiento que se construye.

\footnotetext{
Recordemos aquí la advertencia de Cervo (2003, p. 5) de que las “teorías ajenas” cuando son “epistemólogicamente inadecuadas” pueden ser "políticamente nocivas".

6 Este concepto describe el proceso por el cual el movimiento indígena en América Latina unió ambos aspectos, frente a los peligros de folcklorización que tenía la acentuación de lo étnico y de pérdida de identidad que tenía la acentuación de lo político. Por medio de él, la praxis política y la producción de conocimiento propio quedaron indisolublemente unidas y en interacción dialéctica por el cual se enriquecen mutuamente los dos aspectos.
} 
La primera fuente son las sabidurías indígenas y populares, con un lugar destacado en estas últimas para las sabidurías campesinas. La experiencia de las luchas por la vida, la justicia y la liberación en nuestros pueblos es la base de la elaboración de una riqueza sapiencial. Orlando Fals Borda (2009, p. 279) sostenía que "es fundamental conocer y apreciar el papel que juega la sabiduría popular, el sentido común y la cultura del pueblo, para obtener y crear conocimientos científicos". Esta experiencia de lucha abarca desde las manifestaciones más expresamente políticas y públicas hasta las que en el silencio cotidiano realizan las familias trabajadoras.

La segunda fuente, íntimamente unida a la anterior, es la reflexión sobre nuestra historia regional hecha desde $a_{b a j o}{ }^{7}$.Aquí tiene lugar un proceso dialéctico entre la memoria y la utopía. Se comprende y se reconstruye la historia desde lo que aportan los investigadores con perspectiva popular y desde la memoria ancestral. Desde la memoria de las luchas populares se la comprende como búsqueda de horizontes liberadores (Dussel, 1983, p. 314). En esta dialéctica la memoria sitúa la utopía.También la sitúa en cuanto a las potencialidades de construcción de la utopía en una lectura realista basada en la experiencia popular. La utopía tiene un lugar en esta memoria popular, en esta memoria desde abajo, por eso es más bien —cometiendo el atrevimiento de mezclar el latín con el griego- una subtopía. Un horizonte existente, pero no alcanzado plenamente, hacia el cual se camina. Toda esta dialéctica es, a la vez, política y epistémica.

La tercera fuente está en los autores originales latinoamericanos. La originalidad tiene que ver con una capacidad creativa enraizada en ese subsuelo social del que hablaba Kusch. Es original por ser creativa, ni calco, ni copia, como diría Mariátegui, por ser “creación heroica” (2005, p. 452). Y es original, también, por estar basada, por lo menos en última instancia, en la sabiduría de los pueblos originarios de nuestra región. Por eso, no solamente se trata de autores que nacieron o trabajaron en nuestra región, se trata de una actitud intelectual de los que buscaron y crearon desde nuestros pueblos, desde nuestras realidades y desde un compromiso epistémico y político con las luchas populares. Son muchísimos, pero generalmente fueron excluidos o invisibilizados por los poderes políticos y por la academia hegemónica. Los dos que nombré arriba (Mariátegui y Kusch) son buenos ejemplos de ello.

Si analizamos los contenidos de nuestra academia, y muy especialmente la de Relaciones Internacionales, vamos a encontrar un profundo desconocimiento de esas tres fuentes (Fals B. y Mora O., 2004, p. 2). Es una de las razones de la tendencia a copiar el pensamiento hegemónico. Entre otras cosas porque eso sirve de refugio seguro en las carreras académicas. Salir de ese refugio da miedo al que desconoce esas otras fuentes. Esa es una de las causas más fuertes del eurocentrismo y conservadurismo académicos. Esto, además de expresar la colonialidad del saber, nos muestra la dimensión de la tarea por realizar. Una de las más importantes es lograr una actitud de discipulado de la academia frente a los saberes populares. En la descripción que hace Dussel (1974, pp. 195-196) de la filosofía latinoamericana dice que "es el pensar que sabe escuchar discipularmente la palabra [...] del oprimido, que sabe comprometerse [...] en la movilización de la liberación, y en el mismo caminar [...] va accediendo a la interpretación precisa de su significado histórico".

Hobson y Sajed (2017, pp. 55I-552) rescatan esta idea para las Relaciones Internacionales referenciándose en Edward Thompson, Dipesh Chakrabarty, Rajib Guha y los estudios subalternos. 


\section{La teoría de las Relaciones Transestatales sostiene que la soberanía popular debe ser distinguida y recolocada por encima de la soberanía estatal y, por esto, ser su guía y referencia y que para fortalecer ambas dimensiones de la soberanía en América Latina y el Caribe se tienen que buscar formas de ejercer una soberanía compartida entre pueblos y estados de la región}

En casi todos los países del mundo se invoca a la soberanía popular como fuente de la creación de los estados nación, cuya institucionalidad estaría al servicio de aquella. Sin embargo, la palabra soberanía fue recayendo cada vez más y en forma absoluta sobre el estado. Una de las mediaciones de este traspaso lo hizo el concepto de soberanía nacional entendida en el marco monolítico del estado nación moderno.

Por eso la teoría de las Relaciones Transestatales propone, en primer lugar, resignificar el contenido del concepto de soberanía popular desde las autodeterminaciones primeras que nuestros pueblos necesitan y desean: la alimentaria, la de la salud y la de los saberes, que incluye la educación. Por eso son componentes nucleares de la soberanía popular: la soberanía alimentaria, la soberania sanitaria y la soberanía de saberes.

Aquí es necesario también un estudio profundo y una recuperación del sentido del concepto de soberanía popular utilizado sobre todo en el proceso independentista. Sobre todo en los líderes que se referenciaron en un apoyo claramente popular aparece este sentido de soberanía. José Gervasio de Artigas, Gaspar Rodríguez de Francia, Miguel Hidalgo y José María Morelos tenían una referencia en este tema mucho más hacia su contenido suareciano ${ }^{8}$ —en el que ellos y los pensadores que los acompañaban habían sido educados políticamente- que en los contenidos que venían de Francia (Rousseau fundamentalmente) y Estados Unidos (Jefferson sobre todo). Con la conformación de los estados nación — realizada principalmente en la segunda mitad del siglo $\mathrm{XX}$ - la soberanía popular es postergada en su consideración real y se va sometiendo a lo que se da en llamar soberanía nacional. Así el concepto de soberanía quedó unido a la idea de control sobre un territorio determinado e identificado con el imaginario de estado/nación. Esta distorsión es una de las razones para comprender la lejanía de las teorías de las Relaciones Internacionales de temas centrales de la vida de los pueblos latinoamericanos (Ruiz Briceño y Simonoff, 2017, p. 5I). Una de esas ausencias es el tratamiento de la pobreza (Cimadamore, 2008, p. 18).

En segundo momento, se propone retomar la primacía de la soberanía popular sobre la soberanía estatal. Recordando que "la soberanía es de la comunidad política, del pueblo —no del estado_-" (Dussel, 2006, p. I02). Este contenido se revitalizó con la emergencia de los movimientos populares que enfrentaron al neoliberalismo en nuestra región (Álvarez, 20I3). Desde allí ha sido recuperado también en el nuevo constitucionalismo latinoamericano (Serbin, 2010, p. 2I).

Desde esta revalorización y reposicionamiento del concepto, un tercer momento que está dado por la resignificación del concepto de soberanía compartida, utilizado por gobiernos

\footnotetext{
Recordemos que en la doctrina neoescolástica populista de Francisco Suárez (I548-I6I7) el pueblo es el sujeto del poder político lo cual le da el derecho de luchar contra cualquier tiranía. Esta doctrina que fue oficilizada por la orden jesuita como su teología política oficial fue enseñada en las universidades latinoamericana y estuvo presente en la formación de buena parte de los líderes independentistas. Ella perduró un buen tiempo aún después de la expulsión de la orden de la región.
} 
neoliberales (Gavião y Saraiva, 2019, p. 66) para justificar la intromisión de potencias en el marco de la globalización. Pensado desde la primacía de la soberanía popular sobre la estatal, se abren caminos para una soberanía plurinacional latinoamericana y caribeña. Poder compartir nuestras soberanías nos podría dar mucha más soberanía si esto se hace en la línea de las soberanías populares, si se hace en relación a las expectativas de justicia de nuestros pueblos. Decía el excanciller brasileño Celso Amorín que "la soberanía solamente puede ejercitarse plenamente con una efectiva cooperación entre países similares como son los nuestros” (Brieger, 2020).

\section{La teoría de las Relaciones Transestatales sostiene como necesario en su construcción de conocimiento un análisis del racismo de clase que atraviesa las llamadas Relaciones Internacionales}

Como lo planteara Aníbal Quijano (20/4), el patrón de poder mundial, inaugurado en América Latina está marcado por una clasificación humana que intenta naturalizar la dominación capitalista la cual une las diferenciaciones binarias de clase y raza. Este patrón de poder opera, también, en las relaciones que trascienden a los estados. El sujeto clave en esta operación son los sectores de poder que tienen una visión clasista de las sociedades y que procesan su naturalización a través del racismo.

Dentro de la misma academia estadounidense se viene planteando que las teorías de las Relaciones Internacionales están marcadas por un racismo oculto con una ideología de darwinismo social al servicio de una dominación supremacista blanca (Henderson, 2013, p. 3). Ellas justifican y alientan un sistema taxonómico de la humanidad otorgando a las razas "inferiores" un status epistemológico de silencio e invisibilidad (Persaud y Walker, 200I, p. 374).

Planteo la necesidad de trabajar en la construcción de un análisis de racismo de clase pensado desde América Latina y el Caribe, pero con elementos que pueden ser útiles para otros lugares. A la vez que se pretende afirmar la validez del análisis de clase para nuestra realidad, se propone trabajarlo desde nuestra historia y desde nuestra realidad en diálogo con la propuesta de la colonialidad del poder.

El concepto de racismo de clase fue trabajado por Étienne Balibar (I99|,p.326) quien entendía su función como la de "marcar con signos genéricos poblaciones destinadas colectivamente a la explotación capitalista”. Quijano (20I4) sostiene que se construyó una naturalización de la dominación explotación colonial capitalista utilizando el concepto inventado de "raza" y que esto dio eficacia y perdurabilidad a la producción de "una sistemática división racial del trabajo".

Hacía tiempo que Rodolfo Stavenhagen (1996, p. 20) había planteado que el análisis de clase era un instrumento metodológico desarrollado en los países occidentales para sus sociedades -y más restringidamente para ámbitos industriales y urbanos- pero que había pocos esfuerzos sistemáticos para situar y enriquecer estos análisis en América Latina. Generalmente tomó un carácter de método heredado que es necesario superar para que pueda ser repropuesto en forma situada e historizada desde la realidad latinoamericana.Así podría dar lo mejor de sí en una construcción epistémico política regional. 
Este enriquecimiento puede fortalecer el carácter dinámico y estructural del análisis de clase (Stavenhagen, 1996, p. 26). Al dialogar con la colonialidad del poder se incorporarían categorías históricas propias dándole claridad al vínculo con la realidad regional y quitándole cualquier tendencia mecanicista. Además, se fortalecería la relación entre los conceptos claves de dominación y explotación (Miliband, 1995, pp. 421-423) o expresado de otra forma de poder y clase. En la interpretación de Quijano que realiza César Germaná (2010, p. 213) sostiene que al relacionar este autor la heterogeneidad estructural con la dependencia logra describir la estructura de poder latinoamericana como una asociación de explotación y dominación lograda por la articulación de los sectores dominantes de los estados nación con los de nivel internacional. Por último, sobre todo desde el concepto de raza, se incorporarían otros factores movilizadores enriqueciendo la explicación de la motricidad de la lucha de clases en la región.Aquí está presente el aporte que buscó Mariátegui al resaltar la importancia indígena en esta lucha.

Este enriquecimiento del análisis de clase desde el planteo de racismo de clase puede servir también para todas las sociedades marcadas por la colonialidad, lo cual abarca todo el Sur Político, pero también las marcas de esta colonialidad en el Norte hegemónico como se ve claramente con las explosiones populares contemporáneas luego del asesinato de George Floyd. El planteo tiene la ventaja de no diluir la visión antagónica del análisis social y, a la vez, de enriquecerlo histórica y culturalmente, posibilitando una mirada más clara a la dimensión epistémico política que posibilita pensar la producción de conocimiento desde la praxis de la lucha popular?

El tratamiento de este tema está ligado al de la heterogeneidad estructural.Tradicionalmente tratado desde la visión cepalina, puede resignificarse desde la visión mariateguiana de nuestras formaciones sociales, como lo señala Quijano (2014, p. 288). Desde esta visión el análisis no solamente se enriquece, sino que también, en cierta medida, se revierte. Porque la potencialidad epistémica, política y económico ecológica de las partes consideradas habitualmente como atrasadas y cómo rémoras para el desarrollo, las convierten en una alternativa para pensar un modelo propio para América Latina. La tradicional negatividad se transforma —al decir de Dussel - en "positiva exterioridad". La propuesta del Buen Vivir es una muestra clara de eso.

También es importante aquí, un diálogo entre la dicotomía clasista (clase burguesa y clase obrera) con lo que podemos Ilamar la dicotomía populista (oligarquía y pueblo). Este diálogo es importante a nivel teórico, pero también a nivel práctico en la construcción de unidad de las fuerzas populares. El concepto de clases populares puede ser útil en este diálogo:

"La identidad del sujeto colectivo pueblo es heterogénea en sus elementos constitutivos, y homogénea en su enmarcamiento en el mundo de la pobreza y en su confrontación con la explotación y la opresión —si bien las manifestaciones de esa confrontación asumen una amplia variación-. La pluralidad de elementos constitutivos obliga a referirse a las 'clases populares' como sujeto doblemente colectivo (por la heterogeneidad de sus ingredientes y por sus expresiones),

Existe un potencial de vinculación de este planteo con las teorías marxistas de la dependencia que tuvieron a Ruy Mauro Marini y Vania Bambirra como figuras centrales, aunque estos autores no vincularon con claridad los temas de clase con el racismo. 
donde el concepto de clase abandona su referente estrecho al trabajador: I) productivo, 2) asalariado y 3) del mercado formal, para englobar a todos quienes participan como explotados $y$ oprimidos en las relaciones de poder político, económico, de género, cultural, étnico" (Vilas, 1995, p. I5).

También aquí el concepto de pobre debe ser considerado, desde su valoración epistémico política en la Filosofía y la Teología de la liberación, sobre todo entendiéndolo como corazón del pueblo y como referencialidad básica de lo popular. Es un elemento clave para la situacionalidad y popularización de una mirada de clase latinoamericanizada.

\section{La teoría de las Relaciones Transestatales pretende aportar conocimiento que dé consistencia y validez al proceso de integración contrahegemónica de la región latinoamericana y caribeña}

Aunque sería preferible - ética e históricamente - hablar de unión latinoamericana y caribeña, utilizo el término integración para describir procesos de articulación regional con la intención de participar en el debate político y de las llamadas Relaciones Internacionales. Lo hago desligándolo de su vínculo histórico con las teorías del desarrollo, su consecuente economicismo; de la impronta europea que tiene en su tratamiento académico, y de su utilización intraestatal para describir procesos de incorporación desde arriba al orden capitalista de los más victimados y empobrecidos, principalmente los pueblos indígenas. Me refiero aquí a procesos de unión transestatales respetuosos e igualitarios.

El término gana en claridad y referencialidad política al calificarlo como contrahegemónico. Históricamente los pasos concretos y las reflexiones más valiosas fueron y son en este sentido. Fueron realizados cuando contenían una dimensión liberadora de las acciones imperialistas en la región. Y en los últimos dos siglos fue clave —en forma más confrontativa o más diplomáticala superación de la política dominadora de Estados Unidos. Ya advertía Mariátegui (2005, p. 45I) que "lo que separa y aisla a los países latinoamericanos" es que "funcionan como colonia de la industria y la finanza europea y norteamericana". Décadas después se reafirmaba esto: "Estados Unidos representa la fuerza extrarregional que obstaculiza la integración” (Puig, 1978, p. 96). Los distintos intentos y procesos de unión latinoamericana tuvieron esta impronta, fueron siempre una respuesta a "amenazas externas" (Briceño Ruiz, 20I4, p. 14) y los de los últimos veinte años — con marchas y contramarchas, límites y ambigüedades - fueron importantes en este sentido ${ }^{10}$.

Una de las particularidades de los pasos integradores recientes es que constituyeron el único proceso regional que propuso una contestación al neoliberalismo que domina la mayor parte del planeta. En consonancia con esto se crearon espacios de integración que excluyeron a Estados Unidos (Serbín, 2010, p. 17). Con la llegada al poder de una serie de gobiernos de derecha en los últimos años, este proceso fue seriamente afectado. Se desarticularon, paralizaron y minimizaron

\footnotetext{
10 La amenaza imperial tuvo distintas caras. En el siglo XIX, con varios proyectos imperialistas en pugna, fue francesa en Haití; española en Cuba, Puerto Rico y la guerra del guano; inglesa de una forma más abarcativa y principalmente económica en casi toda la región; pero, desde la afirmación de la hegemonía estadounidense en el período entreguerras, fue y es fundamentalmente relacionada a ésta.
} 
las instituciones construidas para la integración. Algunos interpretaron esta coyuntura como un fin de ciclo populista, pero entiendo que la situación debe analizarse, más bien, en el contexto de un empate catastrófico. La situación de empate se evidencia en la mayoría de los resultados electorales en la región en los últimos diez años donde predomina una partición en dos mitades del apoyo entre bloques de derecha y de izquierda. Esta situación tiene una explicación compleja, pero uno de sus elementos fuertes fue una cantidad de golpes de estado perpetrados en Honduras (2009), Paraguay (20I2), Brasil (2016) y Bolivia (2019) que, a la vez que evidencian cierto retroceso de los bloques populares, también muestran el valor que estos procesos tenían para merecer ser objetos de planes orquestados para derribarlos con evidente apoyo internacional. A esto se suman los cercos creados - más allá de los méritos o deméritos de sus gobiernos - en Venezuela y Nicaragua y la renovación del histórico cerco a Cuba. En todos estos procesos está en juego la acción hegemónica de Estados Unidos con la ruptura del proceso de integración como uno de sus objetivos claves. El hecho de conceptualizar la coyuntura como empate implica que no hay una definición clara en el rumbo regional, incluyendo su proceso de integración. De cualquier forma, la importancia de una lectura profunda del periodo reciente es clave para evaluar límites y potencialidades del proceso de integración en los tiempos actuales. El trabajo para revitalizar la Comunidad de Estados Latinoamericanos y Caribeños (CELAC) a partir de la presidencia mexicana que comenzó en enero de 2020 y apoyada en el eje México Argentina; el camino que viene realizando extrainstitucionalmente el reciente Grupo de Puebla; las rebeliones populares contra los gobiernos neoliberales (Haití, Chile, Ecuador, Bolivia, Guatemala, Perú, Colombia, Paraguay) y procesos electorales recientes como los de Bolivia y Ecuador, son elementos para tener en cuenta en una perspectiva de retomada del camino de unión de nuestros pueblos.

En esta línea contrahegemónica también es importante la clarificación del carácter imperialista del proceso que se denomina como "globalización" (Borón, 2004) y, por tanto, el papel de los Estados Unidos en esta perspectiva, ya que "lo que define el curso de la dominación capitalista en la actualidad es la hegemonía de los Estados Unidos, ejercida con un específico carácter imperialista y que se expresa en forma particular respecto aAmérica Latina, históricamente pretendida como su 'patio trasero'” (Rajland, 2005, pp. 30-3I).

El camino de la unión latinoamericana es un camino que solamente se recorre con coherencia y convicción desde una postura crítica al capitalismo y desde la convicción de la necesidad de superarlo:

"Hispanoamérica, Latinoamérica, como se prefiera, no encontrará su unidad en el orden burgués. Este orden nos divide, forzosamente en pequeños nacionalismos. Los únicos que trabajamos por la comunidad de estos pueblos somos, en verdad, los socialistas, los revolucionarios. [...]A Norteamérica sajona le toca coronar y cerrar la civilización capitalista. El porvenir de la América Latina es socialista" (Mariátegui, 2005, p. 464). 
8. La teoría de las Relaciones Transestatales, pensada desde América Latina, puede ser un aporte al diálogo Sur Sur ya que su planteo descolonizador en lo epistémico y lo político apunta a una realidad común del Sur Político

La idea de la transestatalidad como consecuencia de la plurinacionalidad de nuestros países vale con mucha fuerza para todo el Sur Político cuya experiencia colonial marcó la conformación de los estados nación bajo fronteras que no correspondían a la realidad de los pueblos que los habitaban.

En este sentido, el racismo de clase conformado con la invasión a América según el planteo de la colonialidad del poder es una realidad que se extiende a la configuración de todos los países afectados por los procesos de colonización. Ello implica un permanente enfrentamiento entre los espacios no capitalistas autóctonos de raíces comunitarias y el intento constante de expansión capitalista, una continuidad de la acumulación originaria.

Es muy importante relacionar el proceso de articulación Sur Sur con los hechos y reflexiones en torno al proceso histórico que estuvo expresado en términos de "Tercer Mundo", "Periferia" y "No Alineados" (Cairo y Bringel, 20l0, p. 43). De todas formas hace falta "un marco epistemológico propio" para profundizar en esta articulación y la creación de "un paradigma propio, sobre todo en materia de desarrollo" (Ojeda M., 2019, p. 38) para que sea en beneficio de los pueblos. Así, desde la colonialidad, la construcción de pensamiento original debe ser pensada en el contexto del Sur Político y en diálogo con él (Santos, 2002, pp. 367-380). Sus visiones alternativas "desde abajo" tienen mucho que aportar al debate sobre el orden internacional (Blaney e Inayatullah, 2008, pp. 663-664; Ghilarducci, 2020). Esta dimensión Sur Sur funciona como contexto de toma de posición en la dimensión mundial para este planteo político epistémico.

\section{La teoría de las RelacionesTransestatales parte de un compromiso con una búsqueda} de caminos de justicia y de caminos alternativos de superación del capitalismo que partiendo de la praxis y la reflexión del movimiento indígena recupere en un diálogo de saberes fecundo los grandes aportes latinoamericanos en este sentido (teoría de la dependencia, cristianismo de liberación, socialismo indoamericano, y toda la reflexión y la praxis original revolucionaria riquísima de América Latina) tratando de servir así como aporte conjunto, principalmente con las otras regiones afectadas por la colonialidad del poder (o del Sur Político) para que esta búsqueda pueda ser global

En este sentido puede afirmarse que la transestatalidad es fundamentalmente transcapitalista aunque no en igual sentido de trascendencia.Así como la plurinacionalidad implica superar —en principio, fronteras adentro- la actual configuración de los estados nación en cuanto estructurados para servir a la reproducción capitalista, colonial, moderno occidental; la transestatalidad implica superar una configuración de lo que hoy se denomina lo internacional ligada a estos mismos principios. En ambos espacios, estatales y transestatales, la necesidad de pensar desde un planteo Otro, desde la exterioridad - cultural y económica - del sistema dominante es clave. En este contexto cobra relevancia la presentación del Vivir Bien o Buen Vivir "como una propuesta alternativa a la crisis del capitalismo global" (Choquehuanca, 20I3). 
Esto implica una convicción de la necesidad de articulación que supere la tradicional tendencia a fragmentarse de los proyectos populares. E implica también, y aquí entra fuertemente la dimensión epistémica, un diálogo abierto, constante y consciente entre las distintas posturas. Es "la recreación de un nuevo internacionalismo de pueblos en lucha, a través de una auténtica perspectiva de integración popular que sea plural, horizontal, con una clara definición ideológica antineoliberal, anticapitalista, antipatriarcal y antiimperialista" (Asamblea de los Movimientos Sociales, 2009, p. 4). El otro aspecto, es la necesidad de implementación y consolidación del diálogo de saberes de ámbitos académicos con los movimientos populares, incluyendo en este diálogo las Relaciones Transestatales.

\section{La teoría de las Relaciones Transestatales trabaja desde un paradigma biocéntrico, con base en las cosmovisiones y praxis de los pueblos y naciones indígenas, el cual tiene que ser un elemento fundamental para articular las relaciones entre los pueblos y entre los estados}

Las propuestas indígenas que fueron madurando bajo la politización de lo étnico cuestionaron la ruptura moderna entre hombre y naturaleza y lograron expresarse en los principios constitucionales de Ecuador y en la proclamación de los Derechos de la Pachamama. Esto afirmó un paradigma biocéntrico, que venía siendo trabajado teóricamente por la ecología profunda, pero que desde los pueblos indígenas de América Latina y el Caribe ganó en profundidad y significado porque ya no se trata solo de una teoría, se trata de la formulación de una praxis ancestral y, a la vez, existente (Tenesaca, 2013, p. 20). El considerar a la naturaleza como inescindible del ser humano y como sujeto de derechos, supera la visión ambientalista (Zaffaroni, 20I I). Y al entender que la vida se refiere "a las personas, tanto como la de las plantas, animales, cerros o manantiales" (Churuchumbi, 20I4, p. 64) supera también la visión de los ecosistemas (Gudynas, 2009, p. 37). También se plantea una superación del concepto de desarrollo sustentable.Aún desde una perspectiva crítica a todos estos conceptos es necesario establecer un diálogo con ellos por su posicionamiento en la agenda política internacional. Frente al capitalismo, dominante en el sistema mundial,"que vive de sofocar la vida" (Martínez y Acosta, 2017, p. 2930) las relaciones transestatales deben encararse, como se planteara desde la cancillería boliviana, como una “diplomacia por la vida” (Choquehuanca, 20I3).

\section{Conclusión}

A lo largo del trabajo han quedado presentados una serie de conceptos y perspectivas que articulados pueden servir como camino a una teoría de las Relaciones Transestatales. Recuerdo brevemente algunos de ellos. Desde el concepto de plurinacionalidad se propone el de relaciones transestatales para el análisis de las relaciones que trascienden a los estados nación. Como lugar epistémico político desde el cual construir este conocimiento se opta por la praxis de las luchas populares por la vida. Se entiende la integración como protagonizada por los pueblos y como contrahegemónica. Se trabaja desde la búsqueda de un horizonte poscapitalista para el cual se entiende clave la confluencia de todos los planteos políticos populares que la propugnan. Se propone una visión biocéntrica desde la praxis ancestral y existente de los pueblos y naciones indígenas para el abordaje del tema ecológico en las relaciones transestatales. 
Desde estos elementos articulados en camino hacia la teoría se pretende ampliar la agenda de las hoy llamadas Relaciones Internacionales y también abordar los temas habituales y actuales de esa agenda con un sentido popular y descolonizador. En esta agenda tiene un lugar preeminente la integración latinoamericana y la articulación Sur Sur.

Aquí hay muchos elementos que faltan proponer: la democratización de los medios de comunicación social; la desoccidentalización del derecho y el pluralismo jurídico; un feminismo popular y latinoamericano; la transdisciplinariedad y/o indisciplinariedad; las metodologías de investigación descolonizadoras; la espacio temporalidad en espiral superando la unilineal evolucionista; un replanteamiento del estado desde América Latina y el Caribe; etc. Soy consciente de la necesidad de su presencia, pero no están presentadas por los límites propios del artículo y también por los personales en el proceso de investigación en curso.

Espero pueda ser un aporte que sume al esfuerzo político e intelectual que se viene realizando, muchas veces en condiciones adversas, para que el quehacer de producción de conocimiento académico en estos ámbitos quede profundamente ligado a las luchas de los pueblos latinoamericanos y caribeños.

\section{Referencias}

Al-Kassimi, K. (2018). ALBA: A decolonial delinking performance towards (western) modernity - An alternative to development project. Cogent Social Sciences, 4. https://doi.org/I0.1080/233 I I886.20 I8.15464I8

Álvarez, S. T. (20I3). La soberanía estatal en perspectivas contemporáneas: del centro a la periferia y del poder a la resistencia. Cuadernos Americanos, I4I, I47-I 74.

Asamblea de los Movimientos Sociales (2.2.2009). Construyendo la integración desde abajo de los pueblos. Impulsando el ALBA y la solidaridad de los pueblos, frente al proyecto del imperialismo. rebelion.org.

Balibar, É. ( I99I). El ‘racismo de clase’. En Wallerstein, I. y Balibar, É. Raza, nación y clase (pp. 3I3-333). IEPALA.

Blaney, D. y Inayatullah, N. (2008). International Relations from Below. En Reus-Smit, C. y Snidal, D. (Eds.). The Oxford handbook of international relations (pp. 663-675). Oxford University Press.

Borón,A. (2004). Hegemonía e imperialismo en el sistema internacional. En Chomsky, N. et al. (Eds.). Nueva Hegemonía Mundial. Alternativas de cambio y movimientos sociales (pp. 7I-83). CLACSO.

Briceño Ruiz, J. (20 14).Autonomía: genealogía y desarrollo de un concepto. Su relación con el regionalismo en América Latina. Cuadernos sobre Relaciones Internacionales, Regionalismo y Desarrollo, 9(I8), 9-4I.

Briceño Ruiz, J. y Simonoff, A. (2017). La Escuela de la Autonomía, América Latina y la teoría de las relaciones internacionales. Estudios Internacionales, 186, 39-89.

Brieger, P. (I7.I.20I0). Celso Amorim, excanciller brasileño, a propósito de la salida de Brasil de la CELAC: 'La política de Bolsonaro es una sumisión total a la política de Trump'. Entrevista a Celso Amorim. nodal.am.

Cairo Carou, H. y Bringel, B. (20l0). Articulaciones del Sur Global: afinidad cultural, internacionalismo solidario e lberoamérica en la globalización contrahegemónica. Geopolítica(s), I(I), 4I-63.

Cepeda Másmela, C., Duarte Villa, R., Souza, M. y Tickner, A. B. (2016). Los internacionalistas latinoamericanos. Una radiografía actualizada. Foreign Affairs Latinoamérica, 54-6I.

Cervo, A. L. (2008). Inserção Internacional: Formação dos conceitos brasileiros. Saraiva.

Cervo, A. L. (2003). Política exterior e relações internacionais do Brasil: enfoque paradigmático. Revista Brasileira de Política Internacional, 46(2), 5-25.

Cimadamore, A. (2008). Las políticas de producción de pobreza: construyendo enfoques teóricos integrados. En Cimadamore,A. y Cattani,A. (Coords.). Producción de pobreza y desigualdad en América Latina (pp. I 7-40). Siglo del Hombre.

Cox, R.W. (20I4). Fuerzas sociales, estados y órdenes mundiales: Más allá de la Teoría de Relaciones Internacionales. Relaciones Internacionales, 24, I29-I63.

Choquehuanca, D. (2013). Discurso en al Acto oficial de Presentación del Documento 'La Revolución Democrática Cultural y su Política Exterior. Memoria Institucional 2006-20I3'. cancilleria.gob.bo.

Churuchumbi, G. (20I4). Usos cotidianos del término Sumak Kawsay en el territorio Kayambi (Tesis de Maestría). Universidad Andina Simón Bolívar Ecuador. repositorio.uasb.edu.ec.

De la Torre,V. (20I I). La acción colectiva transnacional en las teorías de los movimientos sociales y de las Relaciones 
Internacionales. CONfines, 7(I4), 45-72.

Deves-Valdés, E. (20I3). Cómo pensar los asuntos internacionales-mundiales a partir del pensamiento latinoamericano: Análisis de la teorización. Historia Unisinos, I7(I), 48-60.

Díaz Martínez, K. (20I4). Movimientos Sociales e Integración Regional: el caso de la Articulación de Movimientos Sociales hacia el ALBA. Revista Pueblos y Frontera digital, 8( I6), I57-I85.

Dinerstein,A. C. (20I0). Autonomy in Latin America: between resistance and integration. Echoes from the Piqueteros experience. Community Development Journal, I-I I.

Dussel, E. (1974). Método para una Filosofia de la Liberación. Ágora.

Dussel, E. (1983). Praxis latinoamericana y Filosofia de la Liberación. Nueva América.

Dussel, E. (2006). 20 Tesis de Política. Siglo XXI.

Ellacuría, I. (2005). Los pobres, lugar teológico en América Latina.Archivo Chile/CEME.

Fals Borda, O. (2009). Una sociología sentipensante para América Latina. CLACSO / Siglo del Hombre.

Fals Borda, O. y Mora Osejo, L. (2004). La superación del Eurocentrismo. Enriquecimiento del saber sistemático y endógeno sobre nuestro contexto tropical. Polis. Revista Latinoamericana, 7.

Flores Galindo, A. (2008). Obras Completas. Tomo V. Sur.

Gavião, L. y Saraiva, M. G. (2019).América del Sur en la política exterior brasileña de Lula da Silva. América Latina hoy, $82,63-83$

Germaná, C. (20I0). Una epistemología Otra: el proyecto de Aníbal Quijano. Nómadas, 32, 2 I I-22I.

Ghilarducci, D. (2020). La diplomacia desde abajo: una herramienta teórica para los estudios globales. Colombia Internacional, 102, I I3-138.

Gudynas, E. (2009). La ecología política del giro biocéntrico en la nueva Constitución de Ecuador. Revista de Estudios Sociales, 32, 34-47.

Gullo, M. (2017). Los problemas básicos de las Relaciones Internacionales como disciplina de estudio. Diplomatize, $5(\mathrm{III}), \mathrm{I}-39$.

Henderson, E. (2013). Hidden in plain sight: racism in international relations theory. Cambridge Review of International Affairs. https://doi.org/ I0.1080/0955757I.2012.7/0585

Hobson, J.y Sajed,A. (2017). Navigating Beyond the Eurofetishist Frontier of Critical IR Theory: Exploring the Complex Landscapes of Non-Western Agency. International Studies Review, 19, 547-572.

Kusch, R. (1978). Esbozo de una Antropología Filosófica Americana. Castañeda.

Macas, L. (2005). La necesidad política de una reconstrucción epistémica de los saberes ancestrales. En Dávalos, P. (Comp.). Pueblos indígenas, Estado y Democracia (pp. 35-42). CLACSO.

Mariátegui, J. C. (2005). Invitación a la vida heroica. Fondo Editorial del Congreso del Perú.

Martínez, E. y Acosta,A. (2017). Los Derechos de la Naturaleza como puerta de entrada a otro mundo posible. Direito \& Práxis, 8(4), 2927-2961.

Miliband, R. (1995). Análisis de clases. En Giddens, A. y Turner, J. (Eds.), La teoría social hoy (pp. 4I8-444). Alianza.

Ministerio de Relaciones Exteriores del Estado Plurinacional de Bolivia (20I3). La Revolución Democrática y Cultural y su Política Exterior. Memoria 2006-20 I 3. MREEPB.

Ojeda Medina,T. (2019). Introducción. La Cooperación Sur-Sur en América Latina y el Caribe: balance de una década (2008-20 18). En Ojeda Medina, T. y Echart Muñoz, E. (Coords.). La Cooperación Sur-Sur en América Latina y el Caribe: balance de una década (2008-2018) (pp. I5-40). CLACSO.

Persaud, R. B. y Walker, R. B. (200I). Apertura: Race in International Relations. Alternatives, 26, 373-376.

Puig, J. C. (1978). Derecho internacional americano, nacionalismo latinoamericano y régimen internacional. Mundo Nuevo. Revista de Estudios Latinoamericanos, I.

Quijano, A. (20I4). Cuestiones y horizontes: de la dependencia histórico-estructural a la colonialidad / descolonialidad del poder. CLACSO.

Rajland, B. (2005). La estrategia imperialista hoy.Aspectos políticos de la integración en la actualidad y el papel de los Estados Unidos en su relación con América Latina. En Bauer, A. et al. (Coords.). Seminario 'Rosa Luxemburg, pensamiento y acción por el socialismo. América Latina en el siglo XXI'. Buenos Aires.

Rocha, E. S. y Maso, T. F. (2020). Alteridad: la reinvención de la paz en las Relaciones Internacionales. Revista Crítica de Ciências Sociais, I2I, 5-24.

Santos, B. de S. (2002). A Crítica da Razão Indolente: contra o desperdicio da experiência. Para um novo senso comum. A ciência, o direito e a política na transição paradigmática. Cortez.

Serbín, A. (2010). Regionalismo y soberanía nacional en América Latina: Los nuevos desafíos. Documentos CRIES, I5, 5-27.

Soto Acosta, W. (20I5). Introducción. En Soto Acosta, W. (Ed.). Ciencias Sociales y Relaciones Internacionales: nuevas perspectivas desde América Latina. Escuela de Relaciones Internacionales de la Universidad Nacional / CLACSO.

Stavenhagen, R. (1996). Las clases sociales en las sociedades agrarias. Siglo XXI.

Stédile, J. P., Piñero, J.y Bertoldi, M. (20I4). La integración desde los pueblos. En León, I. (Coord.). La ALBA: el horizonte latinoamericano del Siglo XXI. FEDAERS / ALAI.

Svampa, M. y Pereyra, S. (2009). Entre la ruta y el barrio. La experiencia de las organizaciones piqueteras. Biblos.

Tenesaca Caguana, J. D. (20I3). Proceso organizativo de la Ecuarunari: un análisis desde las Asambleas Plurinacionales 2009-20II. (Tesis de Licenciatura) Universidad Politécnica Salesiana.

Tickner, A. (2003). Seeng IR Differently: Notes from the Third World. Millennium. Journal of International Studies, 32(2), 
295-324.

Tickner, A. B., Cepeda, C. y Bernal, J. L. (2015). Anti-Americanismo, Pro-Americanismo y sentido común en América Latina. Foro Internacional, 22 I (LV).

Ticona Alejo, E. (2016). De la diplomacia colonial a la diplomacia de los pueblos. La Migraña. Revista de Análisis Político, $19,40-45$.

Vilas, C. M. (1995). Actores, sujetos, movimientos: ¿Dónde quedaron las clases? Sociológica, 10(28).

Walsh, C. (2008). Interculturalidad, Plurinacionalidad y Decolonialidad: las insurgencias político-epistémicas de refundar el Estado. Tabula Rasa, 9, |3|-|52

Zaffaroni, E. R. (20 I I). La Pachamama y el humano. Madres de Plaza de Mayo. 


\section{RELACIONES INTERNACIONALES}

Revista académica cuatrimestral de publicación electrónica Grupo de Estudios de Relaciones Internacionales (GERI)

Universidad Autónoma de Madrid, España

https://revistas.uam.es/relacionesinternacionales

ISSN 1699 - 3950

f facebook.com/RelacionesInternacionales

3. twitter.com/RRInternacional

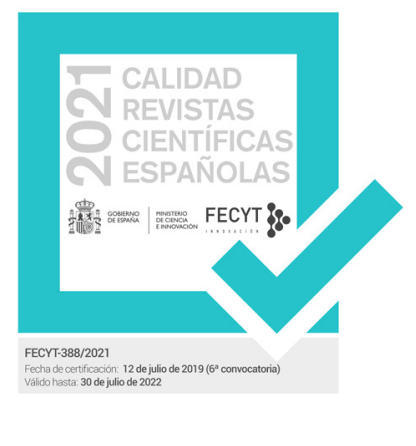

